\title{
SA-I mechanoreceptor position in fingertip skin may impact sensitivity to edge stimuli
}

\author{
Gregory J. Gerling* \\ Department of Systems and Information Engineering, University of Virginia, P.O. Box 400747, 151 Engineer's Way, Charlottesville, \\ VA 22904, USA
}

(Received 28 September 2008; final version received 5 May 2009)

\begin{abstract}
Background: The skin plays a role in conditioning mechanical indentation into distributions of stress/strain that mechanoreceptors convert into neural signals. Solid mechanics methods have modelled the skin to predict the in vivo neural response from mechanoreceptors. Despite their promise, current models cannot explain the role that anatomical positioning and receptor organ morphology play in producing differences in neural response. This work hypothesises that the skin's intermediate ridges may help explain, in part, the sensitivity of slowly adapting type I (SA-I) mechanoreceptors to edge stimuli. Method: Two finite-element models of the fingertip were built, validated and used to analyse the functionality of the intermediate ridges. One of the two-dimensional, cross-sectional models included intermediate ridges, while the other did not. The analysis sought to determine if intermediate ridges (1) increase the magnitude of strain energy density (SED) near the SA-I location and (2) help differentiate one 2.0-mm indenter from two 0.5 - $\mathrm{mm}$ wide indenters with a 1.0-mm gap. Results: Higher concentrations of SED were found near the tips of the intermediate ridges, the anatomical location that coincides with the SA-I receptors. This first result suggested that the location of the SA-Is in the stiffer epidermal tissue helps magnify their response to edge stimuli. The second result was that both models were equally capable of predicting the spatial structure within the in vivo neural responses, and therefore the addition of intermediate ridges did not help in differentiating the indenters. Conclusion: The finding, a 15\%-35\% increase in response when the sampling point lies within the stiffer tissue at the same depth, seeks to inform the positioning of force sensors in robotic skin substrates.
\end{abstract}

Keywords: tactile; finite-element model; SA-I; mechanoreceptor; skin; biomechanics; robotics

\section{Introduction}

Our tactile sense enables the perception of object form, texture and stiffness, which is essential for tasks such as grasping a glass (Johansson 1996). The major underlying physiological mechanisms are well known. Mechanoreceptors in finger-pad skin respond to surface indentation. Each of the four specialised types respond to different stimulus characteristics (Johnson 2001). For instance the slowly adapting type I (SA-I) mechanoreceptors detect static and dynamic deformation of edges, gaps and curvatures. Single receptors convert mechanical forces into trains of neural pulses or action potentials (Vallbo and Johansson 1984). The brain then uses the input from a population of mechanoreceptors to perceive features of the stimulus (Gardner and Kandel 2000; Goodwin and Wheat 2004). Apart from the principles, no one entirely understands how tactile receptors transduce skin deformation into neural signals or how the brain interprets the encoded signals.

One productive way to study mechanoreceptive transduction is to build solid mechanics models capable of reproducing the neural signals observed in vivo (Phillips and Johnson 1981; Maeno et al. 1998; Dandekar et al. 2003; Wu et al. 2004). Specifically, finite-element (FE) models have estimated the stress and/or strain (usually strain energy density or SED) propagating through layered tissue for a given stimulus, in response to the indenters with multiple square gratings. These estimates typically correlate well with the neural inter-spike interval at an $R^{2}$ value between about 0.8 and 0.97 (Phillips and Johnson 1981; Dandekar 1995; Dandekar et al. 2003; Sripati et al. 2006). Although they have yet to do so, such models might help explain the anatomical positioning of SA-I and other receptors and the preferential response for some receptors to static edges versus vibration. Such insights from the human system may help us develop tactile prosthetics and improve robotic interfaces.

Current models, however, tend to represent only macrostructural elements, such as the general curvature of the skin and artificially grouped skin layers. Lack of knowledge of the underlying structures may, in part, restrict the aforementioned questions. For example skin microstructural elements, such as the shape of the interface between the skin layers and receptor depth relative to a stiffness interface, may play integral roles (Gerling 2006). Investigations have begun to examine the impact of skin microstructure. Maeno et al. (1998) relate the microstructure of finger-pad tissue with the locations of the four receptor types Gerling

*Email: gregory-gerling@virginia.edu 
and Thomas (2008) have demonstrated that fingerprint lines may not impact the responsiveness of SA-I receptors.

The present work considers the relationship of the SA-I mechanoreceptors and the intermediate ridges, a skin microstructure at the interface of the epidermis and the dermis, for predicting the response to solid and gap stimuli. This relationship has been noted by researchers who suggest the anatomical proximity may facilitate a functional relationship (Quilliam 1975, 1978; Maeno et al. 1998). Principally, the principal transducer of the SA-I class is the Merkel cell complex (MCC), which includes 5-10 Merkel cells (discs) grouped in a tree-like structure (Guinard et al. 1998). The MCC lies on the tips of the epidermal part of the intermediate ridges at the undulating epidermal-dermal interface. No one knows precisely how the MCC converts mechanical energy into electrical pulses (Mills and Diamond 1995; Ogawa 1996; Tachibana and Nawa 2002; Halata et al. 2003) or why it lies on the bottommost tip of the intermediate ridge (in the basal lamina) (Halata et al. 2003). Perhaps the undulating geometry, relative stiffness (epidermal stiffness is $10-10,000$ times greater than dermal stiffness) or depth from the skin surface may shape the stress propagation to the receptors (Pereira et al. 1991). This work therefore analyses the potential utility of the intermediate ridge - the MCC arrangement.

\section{Methods}

Our two hypotheses are that intermediate ridges help (1) focus SED at the location of the MCC beneath the indenter and (2) modify the shape of SED distributions to create unique spatial structures by which solid and gap stimuli are more distinguishable. To test these hypotheses, numerical experiments compared the distributions of SED generated in FE models with and without intermediate ridges, in response to solid and gap indenters. The two independent variables are the model (ridges or no ridges) and the indenter (one $2.0-\mathrm{mm}$ wide solid or two $0.5-\mathrm{mm}$ wide indenters, with a $1.0-\mathrm{mm}$ gap). The dependent variable is SED.

\subsection{FE model}

Two-dimensional, linear elastic FE models of the cross section of the fingertip were built with and without intermediate ridges. Of the two models, the ridges model has an undulating (sinusoidal) microstructure at the epidermaldermal interface similar to one described by Maeno, except that the model here also includes limiting ridges (Maeno et al. 1998) (Figures 1 and 2). This modification offers more accurate spacing between intermediate ridges. Also, the model's $0.95-\mathrm{mm}$ epidermal thickness is slightly more accurate (Quilliam 1978).

In the no-ridges model, an identical mesh was used, except with changes to the material properties at the epidermal-dermal interface (Figure 2). These changes flatten and extend the epidermal-dermal interface to a con-

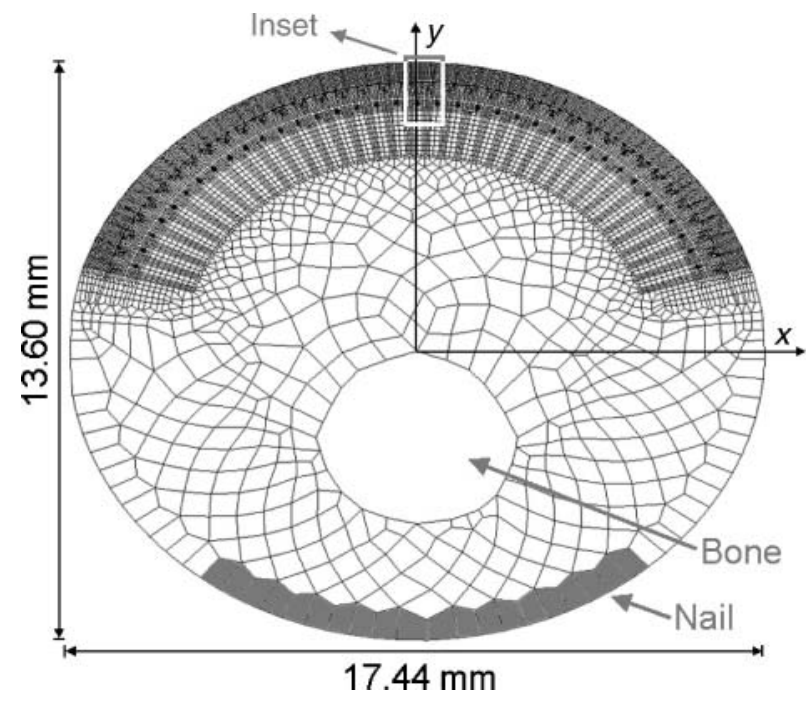

Figure 1. Elliptical model with intermediate and limiting ridges but no exterior papillary ridges, similar to Maeno (Maeno et al. 1998).

sistent depth of $0.7 \mathrm{~mm}$. Several reasons motivated this experimental set-up. First, this strategy preserves an equal volume of epidermis between models and divides the epidermal-dermal interface at the midpoint of the sinusoidal ridges (Figure 2, right inset). Second, it maintains

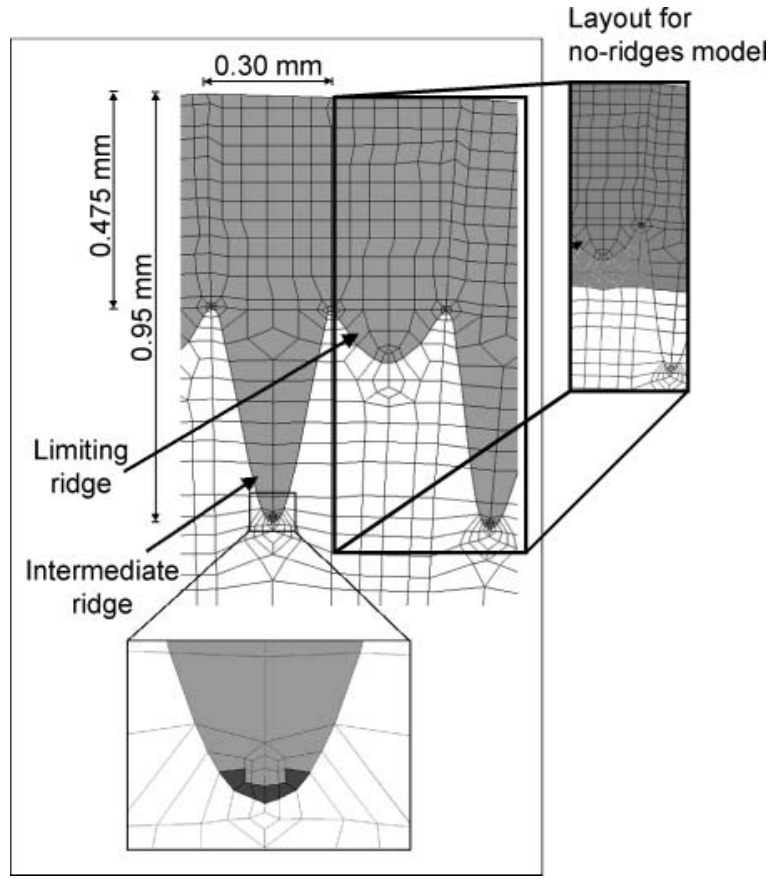

Figure 2. Mesh of the ridges-model section, showing intermediate and limiting ridges. Dark colouring on the lower inset represents the six sampled elements. Element sampling locations were consistent for both models. The right inset represents the material properties settings for the no-ridges model. The same mesh was utilised for both the ridges and no-ridges models. 


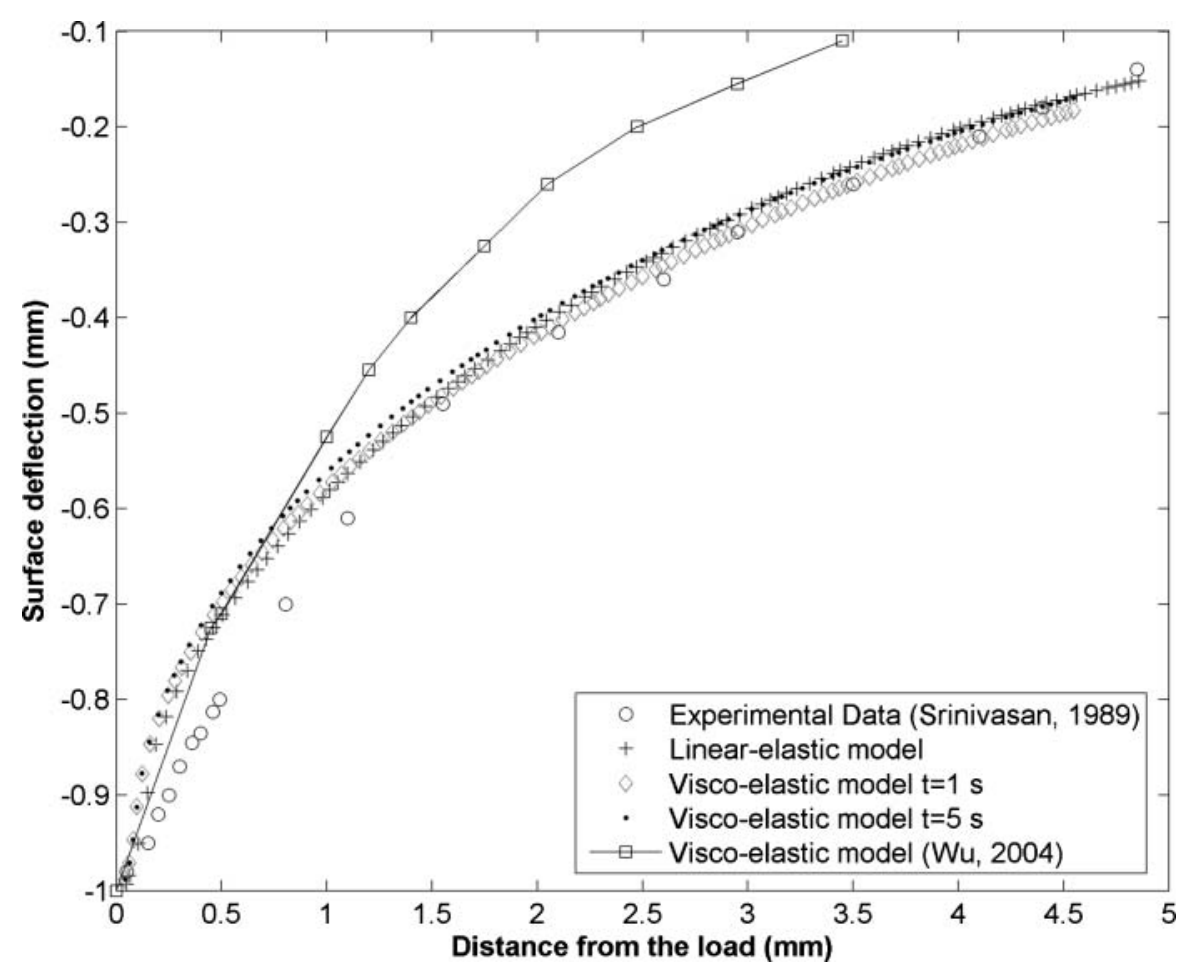

Figure 3. Line load validation test, 50 microns.

the sampling position between the two models (at $0.95 \mathrm{~mm}$ ) but allows for differentiating the impact of sampling in the stiffer epidermis versus that in the more pliant dermis. The strategy of sampling at a specified depth is similar to that employed by others (Phillips and Johnson 1981; Maeno et al. 1998; Dandekar et al. 2003).

The common mesh was built using MSC/PATRAN 2004 software. The entire mesh contains about 16,000 elements and nodes. The mesh is composed of 18 primitives to both left and right of the centre primitive, for a total of 39 per model. The mesh for a single primitive (i.e. one intermediate-ridge section) is shown in Figure 2. Comparatively small elements at the sinusoidal ridge tips permit reasonably square elements. ABAQUS type CPEG4H elements are used. These are four-node, bilinear quadrilateral, hybrid, constant-pressure elements that follow a plane strain assumption (Phillips and Johnson 1981). The material properties used set a Young's modulus of $1.36 \times 10^{5} \mathrm{~Pa}$ for the epidermis, $8.0 \times 10^{4} \mathrm{~Pa}$ for the dermis and $3.4 \times 10^{4} \mathrm{~Pa}$ for the subcutaneous fat with a Poisson's ratio of 0.48 for each layer (Fung 1993; Maeno et al. 1998). While linear elastic material models are often used for small displacements, more sophisticated hyper- or viscoelastic models that take into account the inhomogeneous, nonlinear and anisotropic nature of skin (Agache et al. 1980) may be appropriate for large displacement loads (Ge and Khalsa 2002). Here the assumption of linear elasticity was made based on the validation experiments that employed both linear elastic and hyper-viscoelastic material models. Fixed boundary condi- tions constrain each model at the bone interior and fingernail base. Note that the papillary ridges (exterior fingerprint lines) were excluded because they do not appear to impact the distribution of stress/strain at the SA-I locations at the intermediate-ridge tips (Gerling and Thomas 2008).

Validation of the FE models was done via indentation with a $50-\mu \mathrm{m}$ line load, typical of previous work, whereby predicted skin surface deflection is fitted against that obtained experimentally for the human finger (Srinivasan 1989). Figure 3 indicates that the linear elastic properties used with our model produce a good fit to that experimental data. Note the plotting of the results of Wu's two-dimensional FE model (Wu et al. 2004) that employed hyper- and viscoelastic material properties. These parameters were tested with our model (Figure 3, viscoelastic model $t=1 \mathrm{~s}$ and viscoelastic model $t=5 \mathrm{~s}$ ) but were not used. A second typical validation test seeks to fit stresses and strains calculated near the mechanoreceptor depth location against recorded neural data. The methods surrounding this test are described in Section 2.2, and the results are reported in Section 3.2. Finally, validation of a similar model has been achieved elsewhere (Gerling and Thomas 2008).

\subsection{Numerical tests}

FE software, ABAQUS Standard version 6.6, was used to simulate the indentation of the solid and gap indenters into the ridges and no-ridges models and to estimate the internal distribution of SED. The solid indenter is one $2.0-\mathrm{mm}$ wide 


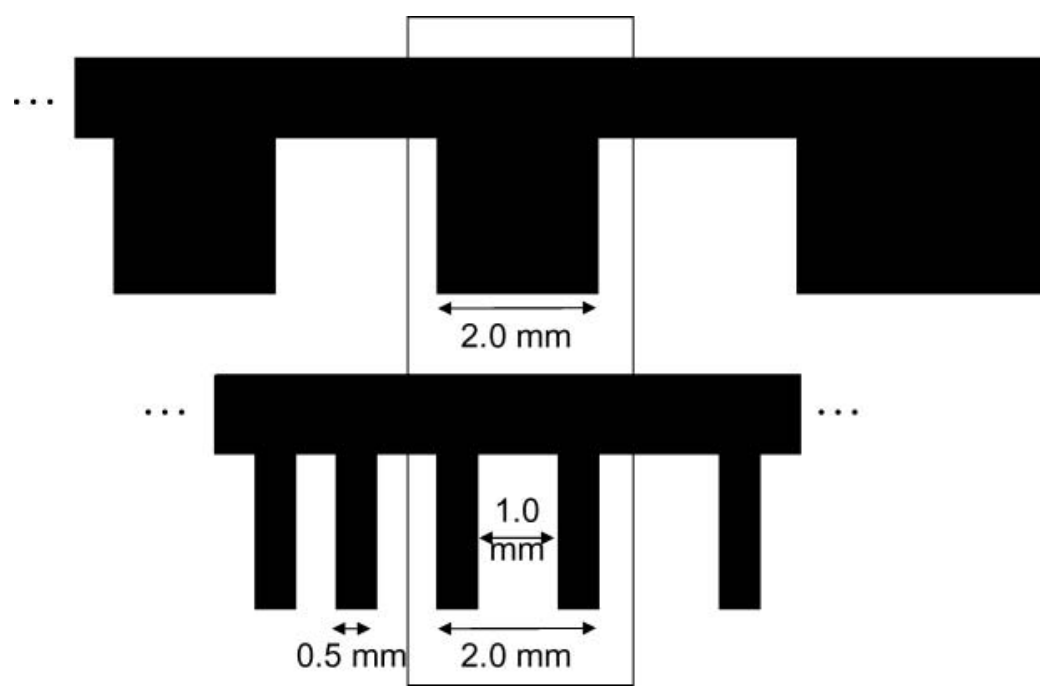

Figure 4. The solid (above) and gap (below) grating indenters. These are sections, indicated by dots at the grating end, of the full indenters in Figure 5. Only these sections of the full indenters made contact with any part of the skin surface. Only those further subsections, indicated by the box outline, made contact with the simulated skin over the sampling point.

indenter, while the gap indenter consists of two $0.5-\mathrm{mm}$ wide indenters, with a $1.0-\mathrm{mm}$ gap between their inside edges (Figure 4). Psychophysical experiments indicate that a gap size of $1.0 \mathrm{~mm}$ is discriminable from a solid bar more than $75 \%$ of the time (Johnson and Phillips 1981; Johnson et al. 2000; Craig and Lyle 2001; Gibson and Craig 2002), in contrast to smaller gap sizes. Therefore we expect the FE model to produce distinct distributions of stress and strain in response to both indenters. Each indenter incorporates a fillet radius of $0.1 \mathrm{~mm}$ at its corners to better approximate a machined metal indenter. These indenters are identical to those used in electrophysiological experiments by Johnson (Phillips and Johnson 1981) (Figure 5).

Each stimulus is displaced to a depth of $1.0 \mathrm{~mm}$ (Phillips and Johnson 1981; Srinivasan and Dandekar 1996; Dandekar et al. 2003; Wu et al. 2004) using a contact interaction. A contact surface is defined between the finger pad

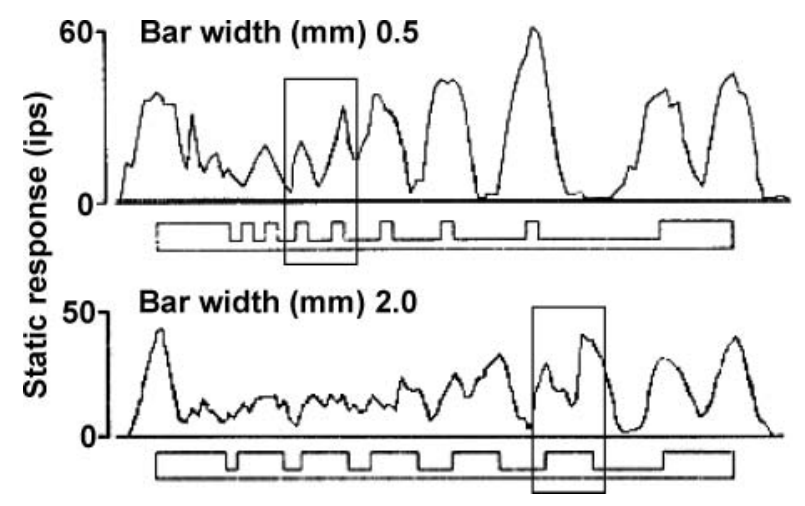

Figure 5. Neural response profiles for two grating stimuli statically imposed on the skin, $0.5 \mathrm{~mm}$ bars (top) and $2.0 \mathrm{~mm}$ bars (bottom). Adapted from Phillips and Johnson (1981). Only the marked areas from each stimulus were of interest in this work. and the indenter with a friction coefficient of 0.3 (Maeno and Kawamura 2003; Gitis and Sivamani 2004). The metal indenter was implemented with an analytical rigid surface. The traditional 'reciprocal interpretation' method was used with multiple adjacent indentations and a single sampling location (Mountcastle and Powell 1959). The indenter was pressed into the simulated tissue surface in separate indentations, spaced in lateral increments of $0.2 \mathrm{~mm}$. SED was measured in selected elements at the single sampling location, where values are averaged for six small elements at a depth of $0.95 \mathrm{~mm}$ on the epidermal side of the tip of the intermediate ridge, centred at the $x$-axis (Figure 2, lower inset). We use here SED as a measure of stress and/or strain near the SA-I location. Our previous work with this model has indicated that SED predicts the SA-I neural response to a 3.0-mm bar indenter well (Gerling and Thomas 2008), and this finding falls in line with others (Phillips and Johnson 1981; Srinivasan and Dandekar 1996; Dandekar et al. 2003; Sripati et al. 2006; Gerling and Thomas 2008). However, these studies also show that maximum compressive strain and maximum tensile strain closely correlate.

By way of definition, the dependent variable $\operatorname{SED}\left(U_{0}\right)$ is comprised of volumetric $\left(U_{\text {ov }}\right)$ and distortional $\left(U_{\text {od }}\right)$ components

$$
U_{\mathrm{o}}=U_{\mathrm{ov}}+U_{\mathrm{od}}
$$

(Gerling and Thomas 2008). However, the volumetric term (hydrostatic pressure $U_{\text {ov }}$ ) is omitted because when Poisson's ratio $(v)$ is set between 0.48 and 0.5 (Srinivasan and Dandekar 1996; Maeno et al. 1998), the material is nearly incompressible and $U_{\mathrm{ov}}$ is indeterminate. The remainder 
then is the distortional stress tensor $\left(U_{\text {od }}\right)$ :

$$
U_{\mathrm{o}}=U_{\mathrm{od}}=\frac{3}{4 G} \tau_{\mathrm{oct}}^{2}
$$

where $\tau_{\text {oct }}$ is the octahedral shear stress and $G$ is the shear modulus of elasticity. Since this is a two-dimensional analysis the normal and shear stresses in the $z$-direction were ignored: ing goodness of fit, where $e_{j}=d_{j}-\varepsilon_{j}$ :

$$
R^{2}=\frac{\sum d_{i}^{2}-\sum e_{i}^{2}}{\sum d_{i}^{2}}
$$

Additionally, in the visualisation contour plots, von Mises stress is shown. For nearly incompressible materials, SED and von Mises stress $\left(\sigma_{\text {yp }}\right)$ are closely related:

$$
\begin{array}{ll}
\tau_{\mathrm{oct}}=\frac{1}{3} \sqrt{\left(\sigma_{x x}-\sigma_{y y}\right)^{2}+\left(\sigma_{y y}-\sigma_{z z}\right)^{2}+\left(\sigma_{z z}-\sigma_{x x}\right)^{2}+6\left(\tau_{x y}^{2}+\tau_{x z}^{2}+\tau_{y z}^{2}\right)}, \\
G=\frac{E}{1+v} . & \text { (4) } \quad U_{\mathrm{o}}=\frac{\sigma_{\mathrm{yp}}^{2}}{G} .
\end{array}
$$

To help test hypothesis 2 (i.e. existence of spatial structure in distributions of SED) the following procedure was used to compare normalised SED $\left(\varepsilon_{j}\right)$ to the electrophysiological recordings of Phillips and Johnson $\left(d_{j}\right)$, using their assumption of linearity (Phillips and Johnson 1981; Srinivasan and Dandekar 1996; Sripati et al. 2006). First the SED was normalised. If the data set $X_{j}$ represents SED, for example, then over the $j$ samples (i.e. indenter location offsets) the data were normalised to $\varepsilon_{j}$ :

$$
\varepsilon_{j}=\frac{X_{j}-\min \left(X_{j}\right)}{\max \left(X_{j}\right)-\min \left(X_{j}\right)}
$$

Then the linear equation $d_{j}=\left(a \times \varepsilon_{j}\right)+b$ was used to correlate the two data sets, where $d_{j}$ is the electrophysiological data (impulses per second) when an indenter is positioned at location offset $j$ and $\varepsilon_{j}$ is the normalised SED under the same condition. Constants $a$ and $b$ were found by maximis-

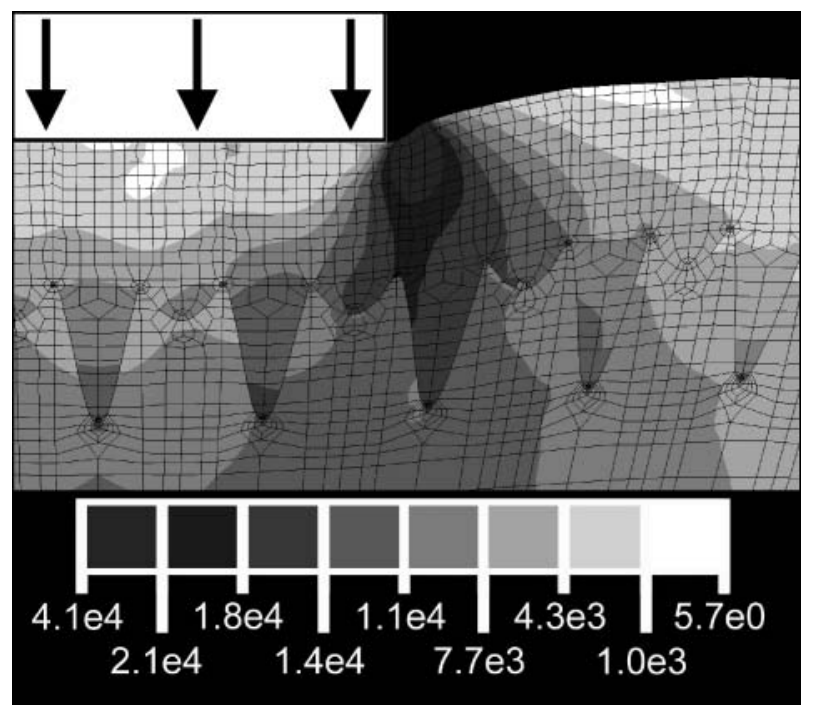

Figure 6. Ridges-model contour plot. Darker shades of grey represent a higher magnitude of von Mises stress. Unit used is $\mathrm{Pa}$.

\section{Results}

The results indicate that the addition of intermediate ridges (1) focuses the SED at the location of the MCCs but (2) does not increase the salience of the spatial structure between the solid and gap stimuli. Therefore, while the ridges model effectively increases the signal gain, both models are adequately capable of discriminating the indenters.

\subsection{Force concentrations at the intermediate-ridge tips}

Figure 6 illustrates that the ridges model concentrates forces at the tips of the intermediate ridges, at the Merkel cell sampling locations. In this contour plot, darker shades of grey

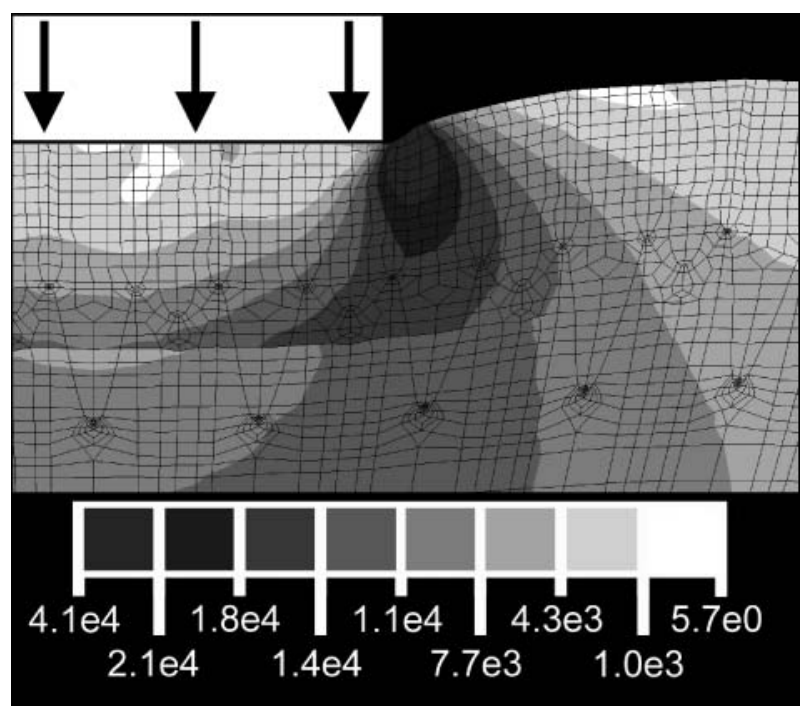

Figure 7. The no-ridges-model contour plot. Note the mesh contained ridges, but the material properties were changed to reflect the flat epidermal-dermal interface at the ridges midpoint. Unit used is Pa. 

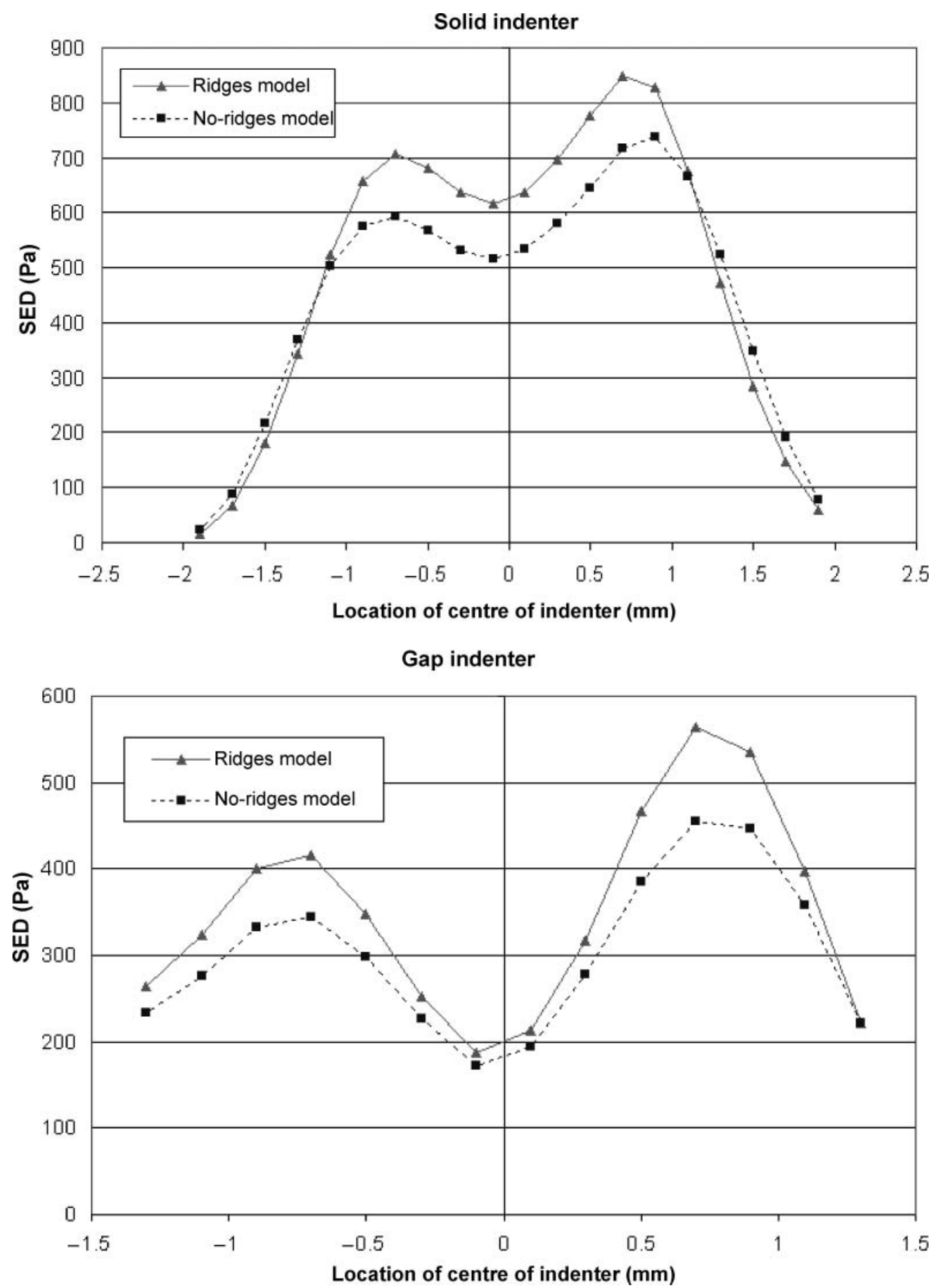

Figure 8. Absolute magnitude of SED measured for the ridges and no-ridges models for the solid (above) and gap (below) indenters.

represent greater magnitudes of von Mises stress. The pattern demonstrated is of a greater magnitude at the epidermal ridge tips than in dermal tissue both beneath the ridge tip and to the left and right of each ridge. For most of the laterally spaced ridges, forces are greater at the ridge tip than elsewhere in the epidermis.
In the no-ridges model in Figure 7, however, von Mises stress is radially dispersed from the stimulus and is not focused toward the sampling-location depth at the tip of the intermediate ridge. Note in viewing the contour plot that except for different material properties for the elements, the no-ridges model's mesh in Figure 7 is identical to the 

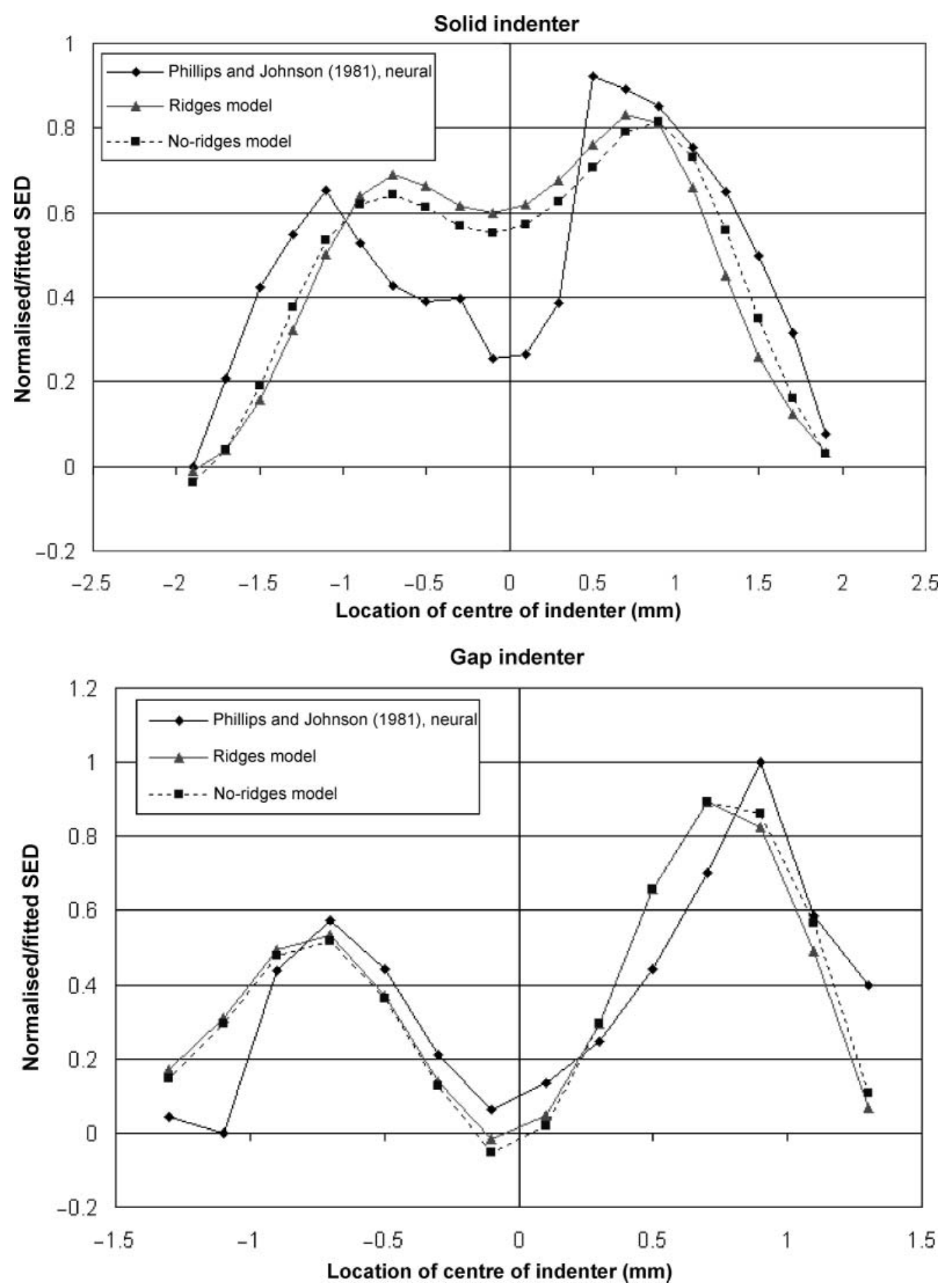

Figure 9. Comparison of measured SED for the ridges and no-ridges models to the neural data of Johnson and Phillips (1981) for the solid (above) and gap (below) indenters as the indenter is stepped over the sampling point.

ridges model's mesh in Figure 6. Figure 7 also indicates the concentration of a greater magnitude of forces on the epidermal side of the epidermal-dermal interface than on the dermal side.

As further illustrated in Figure 8, the absolute magnitude of SED for the ridges model is of greater magnitude than for the no-ridges model, for both the solid indenter (Figure 8, above) and the gap indenter (Figure 8, below). The difference is especially evident at $x$-axis positions from $-1.0 \mathrm{~mm}$ to $+1.0 \mathrm{~mm}$, i.e. when the indenter is contacting the model above the sampling locations. These findings are in line with the results shown in the contour plots. 


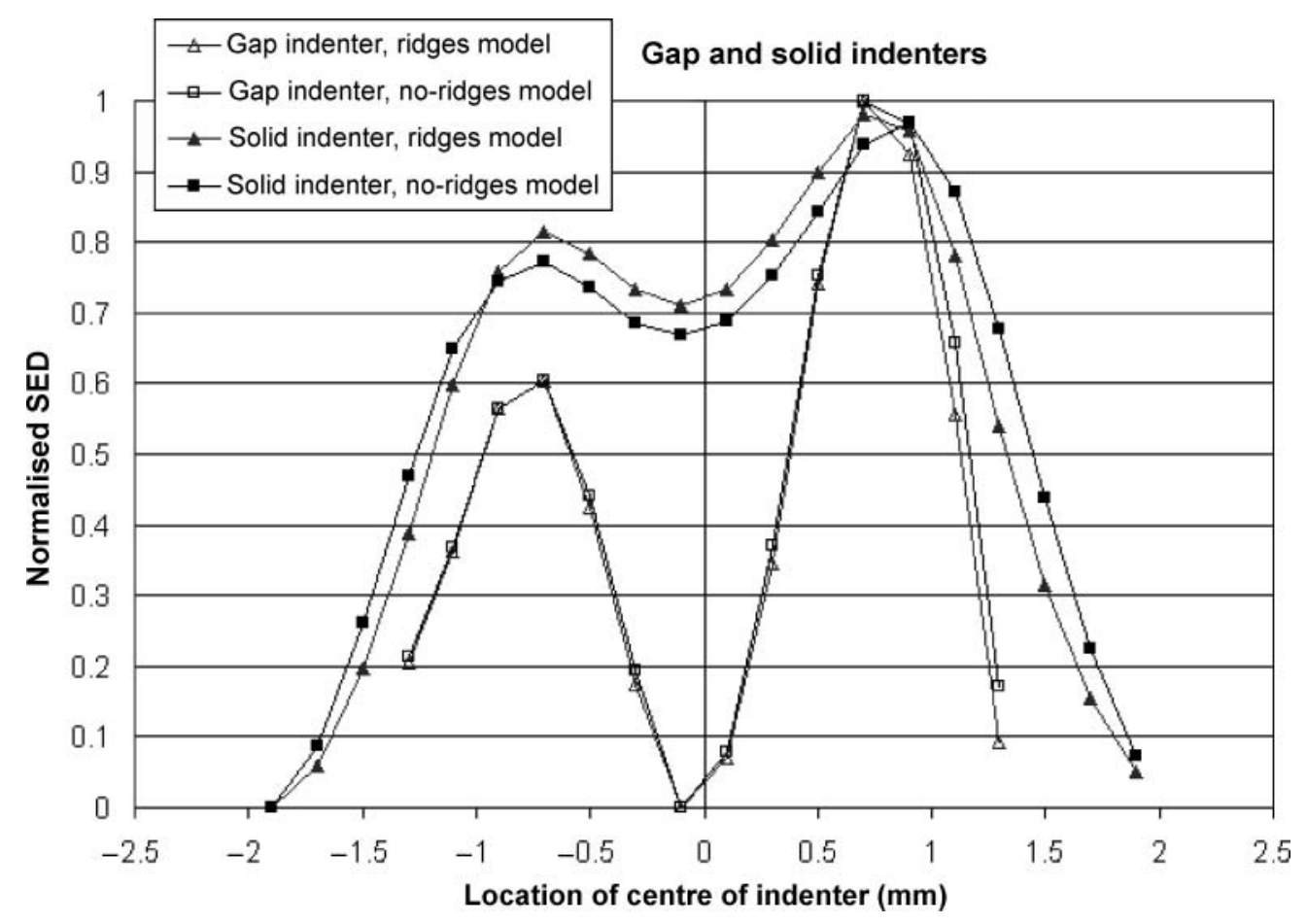

Figure 10. SED predictions normalised and compared for both the models and the indenters.

This difference is quantified by comparing the single SED value closest to $1.0 \mathrm{~mm}$ on the $x$-axis for all four conditions ( 2 models $\times 2$ indenters). The $1.0-\mathrm{mm}$ location is the point at which the indenter's leftmost edge is directly over the sampling location. For the gap indenter, SED is $23.8 \%$ greater for the ridges model than the noridges model. For the solid indenter, SED is $14.8 \%$ greater for the ridges model than for the no-ridges model.

\subsection{Intermediate ridges do not encode spatial structure within SED distributions}

Figure 9 indicates that when the calculated SED is fitted to the neural response, both models achieve an acceptable fit (for the gap indenter $R^{2}$ of 0.85 for both models, for the solid indenter $R^{2}$ of 0.78 for the ridges model and 0.83 for the no-ridges model). Not only are the correlations similar, but they also fall near the predictions of previous models (0.8-0.97) (Phillips and Johnson 1981; Dandekar 1995; Dandekar et al. 2003; Sripati et al. 2006). Normalised SED, in contrast, is displayed in Figure 10. As evident in both figures, once the data are normalised there is little effect due to the model, indicating that the model has little impact in encoding spatial differences between the indenters. That said, both models do clearly produce distributions of SED that adequately differentiate the two indenters.

\section{Discussion}

The results support hypothesis 1 but not hypothesis 2. Including the intermediate ridges (1) focuses the imposed forces to the locations of the intermediate tips, and therefore the sites of the sampling location of the MCC, but (2) does not modify the spatial structure in the SED distributions to make it easier to discern the solid and gap indenters.

For hypothesis 1, the rationale behind the magnified response in the ridges model could arise from its two differences from the no-ridges model: (1) the undulating geometry and (2) the sampling position relative to a change in the material properties (i.e. the sampling position in the ridges model is within the stiffer epidermis, while that in the no-ridges model is within the more pliant dermis) but not due to a different sampling depth. Our previous work, however, has investigated the former and determined that the intermediate-ridge undulations (Gerling 2006), or incidentally the papillary ridges (Gerling and Thomas 2008), do not impact the response. That work, extended here to an elliptical fingertip model verified against the in vivo response, indicates that sampling in the stiffer epidermal tissue is functionally much more relevant than undulating intermediate ridges. In fact, the greatest amplification was found when the sampling position (location of receptors) occurs along a flat interface, on the stiff side of that interface, and when the epidermal thickness is reduced. 

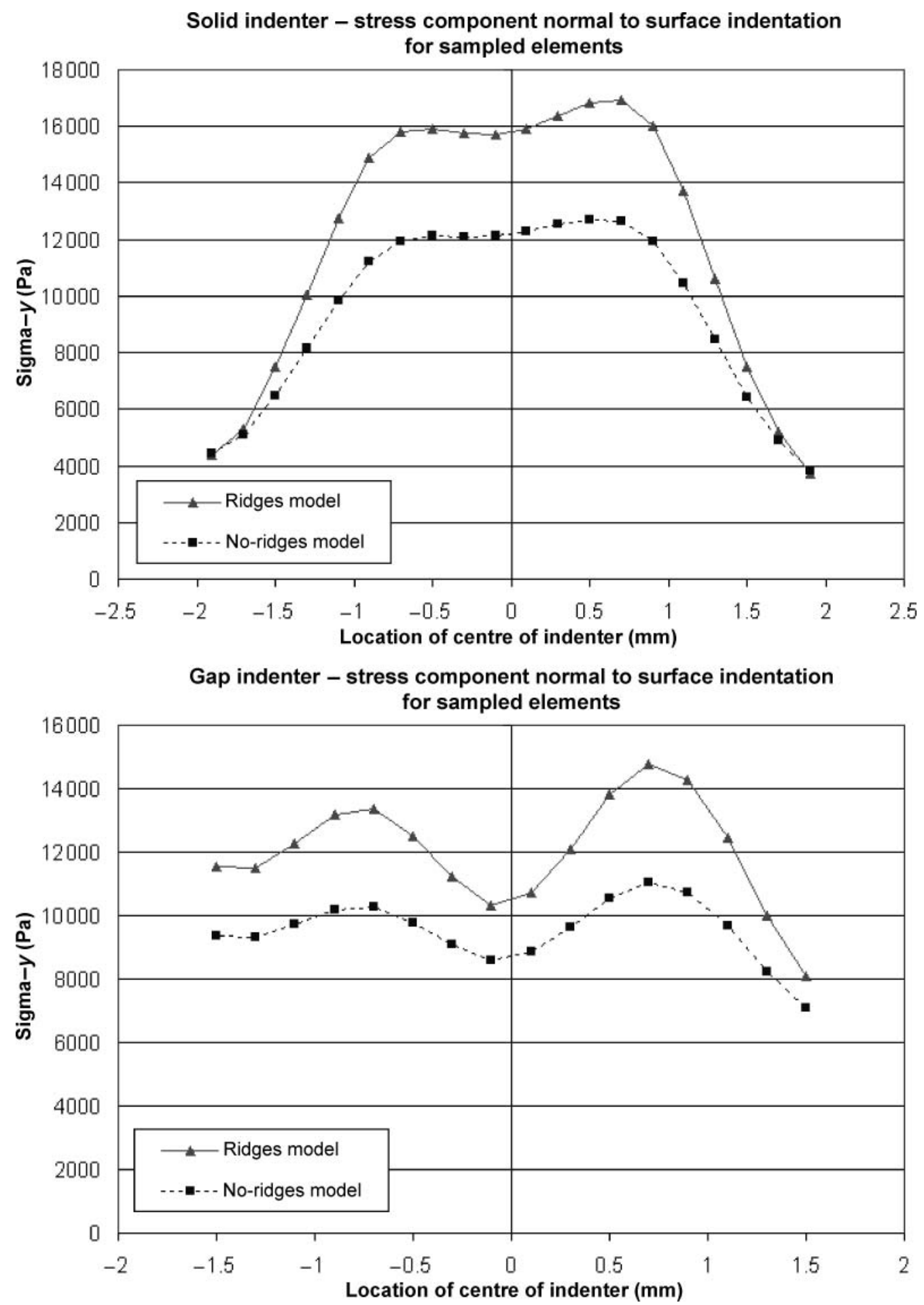

Figure 11. Plots of stress component $\sigma_{y y}$ (i.e. stress in the six sampled elements, in the direction normal to the direction of surface indentation).

This argument - that sampling from the stiffer material is of more utility in magnifying the response than sampling depth - contradicts how previous researchers have tuned their models in the past. Johnson, for example, improved the fit of his model to the in vivo neural response by modifying sampling depth (Phillips and Johnson 1981). The work here suggests the reverse, that the amplitude of response is more sensitive to sampling in the stiffer material than to 
sampling depth. Thus sensor depth could slightly vary with less impact on response. If this holds true in the case of artificial sensors in robotic hands, then the positioning depth of sensors need not be tightly controlled in manufacturing, for a population of tens or hundreds of sensors. Rather one would seek only to insert sensors in the same (preferably upper and stiffer) layer of the elastic substrate.

The explanation for force magnification arising from of sampling in the stiffer material comes straightforwardly from Hooke's law ( $\sigma=E \varepsilon$, where $\sigma$ is stress, $E$ is the modulus of elasticity and $\varepsilon$ is strain). Because the dependent measures here (SED) along with those utilised elsewhere (von Mises stress, maximum compressive stress, maximum compressive strain, shear stress and normal stress) are related to stress (see the equations of Section 2.2), an increase in $E$ will produce an increase in $\sigma$, which is the case for the ridges model (i.e. where stiffer tissue is found the sampling location). Given here for conditions of linear elasticity, a similar statement would hold true for hyperelastic materials with similarly increasing, although more non-linear, stress-strain relationships.

Although demonstrated for the dependent metric SED, an increased response is also found for stress measured normal to the direction of surface indentation $\left(\sigma_{y y}\right)$ (Figure 11). The difference in response for this metric between ridges and no-ridges positioning yields a $34.0 \%$ difference for the solid indenter and a $33.4 \%$ difference for the gap indenter. These differences are larger than for the SED metric (14.8\% and $23.8 \%$ differences, respectively). This quantity is of interest because normal force is presumably detectable by a single-axis sensor that could fit within a small area. For example FlexiForce sensors (Tekscan Inc., Boston, MA, USA) are low cost and detect normal force, although the comparison here is not one-to-one because the sensing area in these sensors is an order of magnitude too large, at present.

Finally, the actual differentiation of signal structure is done by the brain. In fact, the entire process of recovering spatial features from a multitude of neural signals is quite complex and involves overlapping receptive fields, various types of inhibitory interactions, neurons devoted to certain sensory inputs and different levels of processing within the brain (Gardner and Kandel 2000). Nevertheless, regardless of what happens after the receptors encode a neural signal, the potential impact of the intermediate ridges is important because the encoding done by mechanoreceptors is done at the beginning of the entire perceptual process.

\section{Conclusion}

Intermediate-ridge skin microstructure may be one physiological key to tactile edge sensation. To determine the influence of intermediate ridges, two FE models were analysed, with and without ridges. After applying the gap and solid indenters to either model, distributions of SED in- dicated that the ridges model concentrated forces at the intermediate-ridge tips, while the no-ridges model diffused SED throughout its underlying tissue. As a result, the intermediate ridges appear (1) to focus the SED at the location of the MCC beneath the indenter but (2) not to modify the shape of the SED distributions to make the gap and solid indenters easier to discern. The rationale to explain the increased SED response comes from locating the sampling position in a stiffer tissue, in contrast to depth, in a relation that comes from Hooke's law. Increased response of the order of $15 \%-35 \%$ was found and may improve the responses from sensors used in neural prosthesis and robotic sensing.

\section{References}

Agache PG, Monneur C, Leveque JL, Derigal J. 1980. Mechanical properties and Young's modulus of human skin in vivo. Arch Dermatol Res. 269:221-232.

Craig JC, Lyle KB. 2001. A comparison of tactile spatial sensitivity on the palm and fingerpad. Percept Psychophys. 63:33747.

Dandekar K. 1995. Role of mechanics in tactile sensing of shape. $\mathrm{PhD}$ thesis. MIT, Cambridge, MA, USA.

Dandekar K, Raju BI, Srinivasan MA. 2003. 3-D finite-element models of human and monkey fingertips to investigate the mechanics of tactile sense. J Biomech Eng. 125:682-691.

Fung YC. 1993. Biomechanics - mechanical properties of living tissues. 2nd ed. New York: Springer-Verlag.

Gardner EP, Kandel ER. 2000. Touch. In: Kandel ER, Schwartz JH, Jessell TM, editors. Principles of Neural Science. New York:McGraw-Hill. p. 451-471.

Ge WQ, Khalsa PS. 2002. Encoding of compressive stress during indentation by slowly adapting type I mechanoreceptors in rat hairy skin. J Neurophys. 87:1686-1693.

Gerling GJ. 2006. The sampling position within, not the undulating geometry of, fingertip skin microstructure may amplify the sensation of edges. Paper presented at: IEEE Haptic Interfaces for Virtual Environment and Teleoperator Systems, 14th Symposium; 25-26 March 2006; Arlington, VA.

Gerling GJ, Thomas GW. 2008. Fingerprint lines may not directly affect SA-I mechanoreceptor response. Somatosens Mot Res. 25:61-76.

Gibson GO, Craig JC. 2002. Relative roles of spatial and intensive cues in the discrimination of spatial tactile stimuli. Percept Psychoph. 64:1095-1107.

Gitis N, Sivamani R. 2004. Tribometrology of Skin. Tribol Trans. 47:1-9.

Goodwin AW, Wheat HE. 2004. Sensory signals in neural populations underlying tactile perception and manipulation. Annu Rev Neurosci. 27:53-77, 2004.

Guinard D, Usson Y, Guillermet C, Saxod R. 1998. Merkel complexes of human digital skin: three-dimensional imaging with confocal laser microscopy and double immunofluorescence. J Comp Neurol. 398:98-104.

Halata Z, Grim M, Bauman KI. 2003. Friedrich Sigmund Merkel and his 'Merkel cell', morphology, development, and physiology: review and new results. Anat Rec. 271A:225-39.

Johansson RS. 1996. Sensory control of dextrous manipulation in humans. In: Wing AM, Haggard P, Flanagan JR, editors. Hand and brain: the neurophysiology and psychology of hand movements. San Diego (CA):Academic Press. p. 381-414. 
Johnson KO. 2001. The roles and functions of cutaneous mechanoreceptors. Curr Opin Neurobiol. 11:455-461.

Johnson KO, Phillips JR. 1981. Tactile spatial resolution. I. twopoint discrimination, gap detection, grating resolution, and letter recognition. J Neurphysiol. 46:1177-1192.

Johnson KO, Yoshioka T, Vega-Bermudez F. 2000. Tactile functions of mechanoreceptive afferents innervating the hand. $\mathrm{J}$ Clin Neurophysiol. 17:539-558.

Maeno T, Kawamura T. 2003. Geometry design of an elastic fingershaped sensor for estimating friction coefficient by pressing an object. Paper presented at: IEEE International Conference on Robotics and Automation.

Maeno T, Kobayashi K, Yamazaki N. 1998. Relationship between the structure of human finger tissue and the location of tactile receptors. JSME Int J. 41:94-100.

Mills LR, Diamond J. 1995. Merkel cells are not the mechanosensory transducers in the touch dome of the rat. J Neurocytol. 24:117-134.

Mountcastle VB, Powell TP. 1959. Neural mechanisms subserving cutaneous sensibility, with special reference to the role of afferent inhibition in sensory perception and discrimination. Bull Johns Hopkins Hosp. 105:201232.

Ogawa H. 1996. The Merkel cell as a possible mechanoreceptor cell. Prog Neurobiol. 49:317-334.

Pereira JM, Mansour JM, Davis BR. 1991. The effects of layer properties on shear disturbance propagation in skin. Trans ASME. 111:30-35.
Phillips JR, Johnson KO. 1981. Tactile spatial resolution. III. A continuum-mechanics model of skin predicting mechanoreceptor responses to bars, edges, and gratings. J Neurphysiol. 46:1204-1225.

Quilliam TA. 1975. Neuro-cutaneous relationships in fingerprint skin. In: Kornhuber HH, editor. The somatosensory system. Acton (MA):Publishing Sciences Group. p. 193-203.

Quilliam TA. 1978. The structure of finger print skin. In: Gordon $\mathrm{G}$, editor. Active touch: the mechanisms of recognition of objects by manipulation. Oxford:Pergamon Press. p. 1-18.

Srinivasan MA. 1989. Surface deflection of primate fingertip under line load. J Biomech. 22:343-349.

Sripati AP, Bensmaia SJ, Johnson KO. 2006. A continuum mechanical model of mechanoreceptive afferent responses to indented spatial patterns. J Neurophysiol. 95:3852-3864.

Srinivasan MA, Dandekar K. 1996. An investigation of the mechanics of tactile sense using two-dimensional models of the primate fingertip. Trans ASME. 118:48-55.

Tachibana T, Nawa T. 2002. Recent progress in studies on merkel cell biology. Anat Sci Int. 77:26-33.

Ugural AC, Fenster SK. 2003. Advanced strength and applied elasticity. 4th ed. Upper Saddle River (NJ):Prentice Hall.

Vallbo AB, Johansson RS. 1984. Properties of cutaneous mechanoreceptors in the human hand related to touch sensation. Hum Neurobiol. 3:3-14.

Wu JZ, Dong RG, Rakheja S, Schopper AW, Smutz WP. 2004. A structural fingertip model for simulating of the biomechanics of tactile sensation. Med Eng Phys. 26:165-175. 

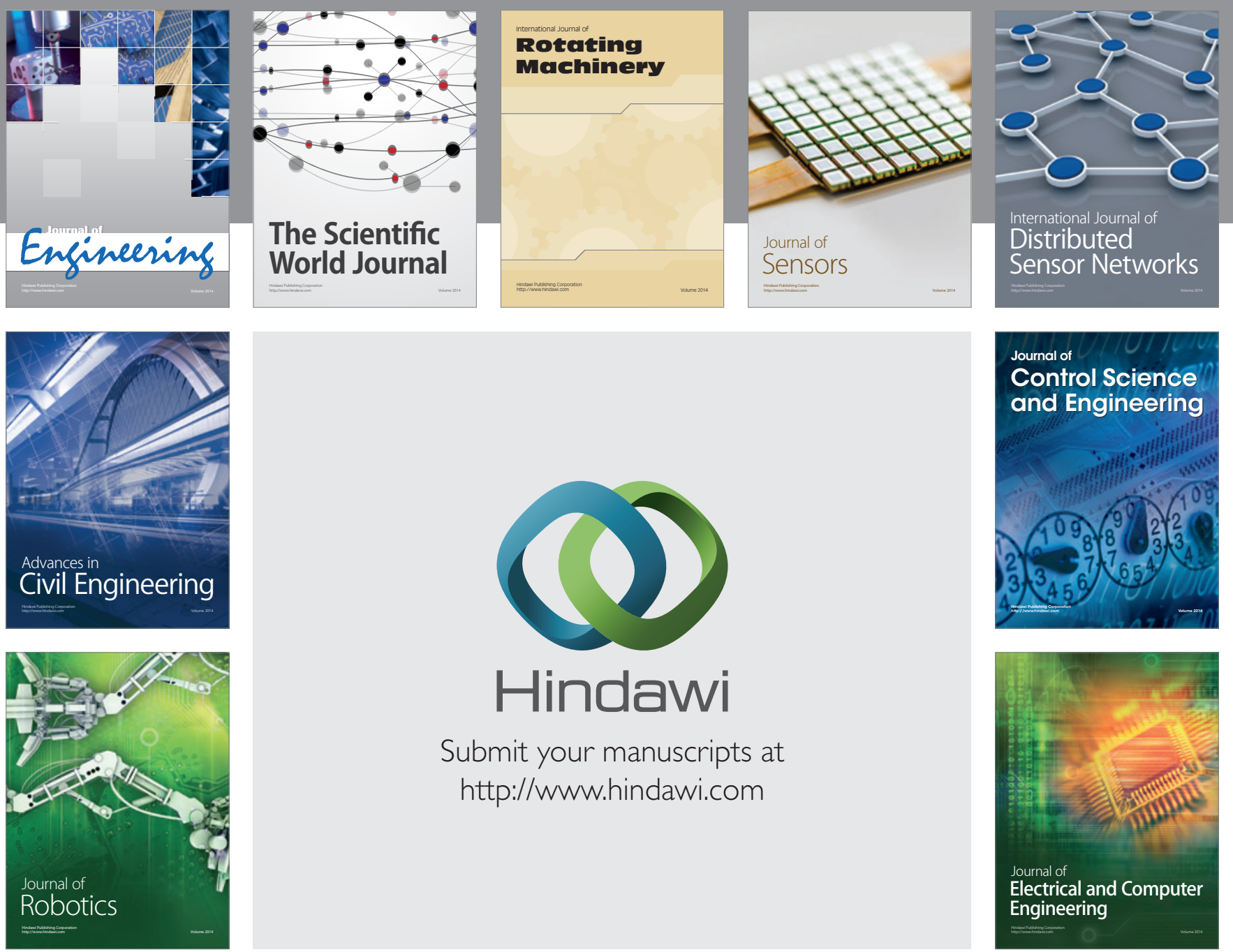

Submit your manuscripts at

http://www.hindawi.com
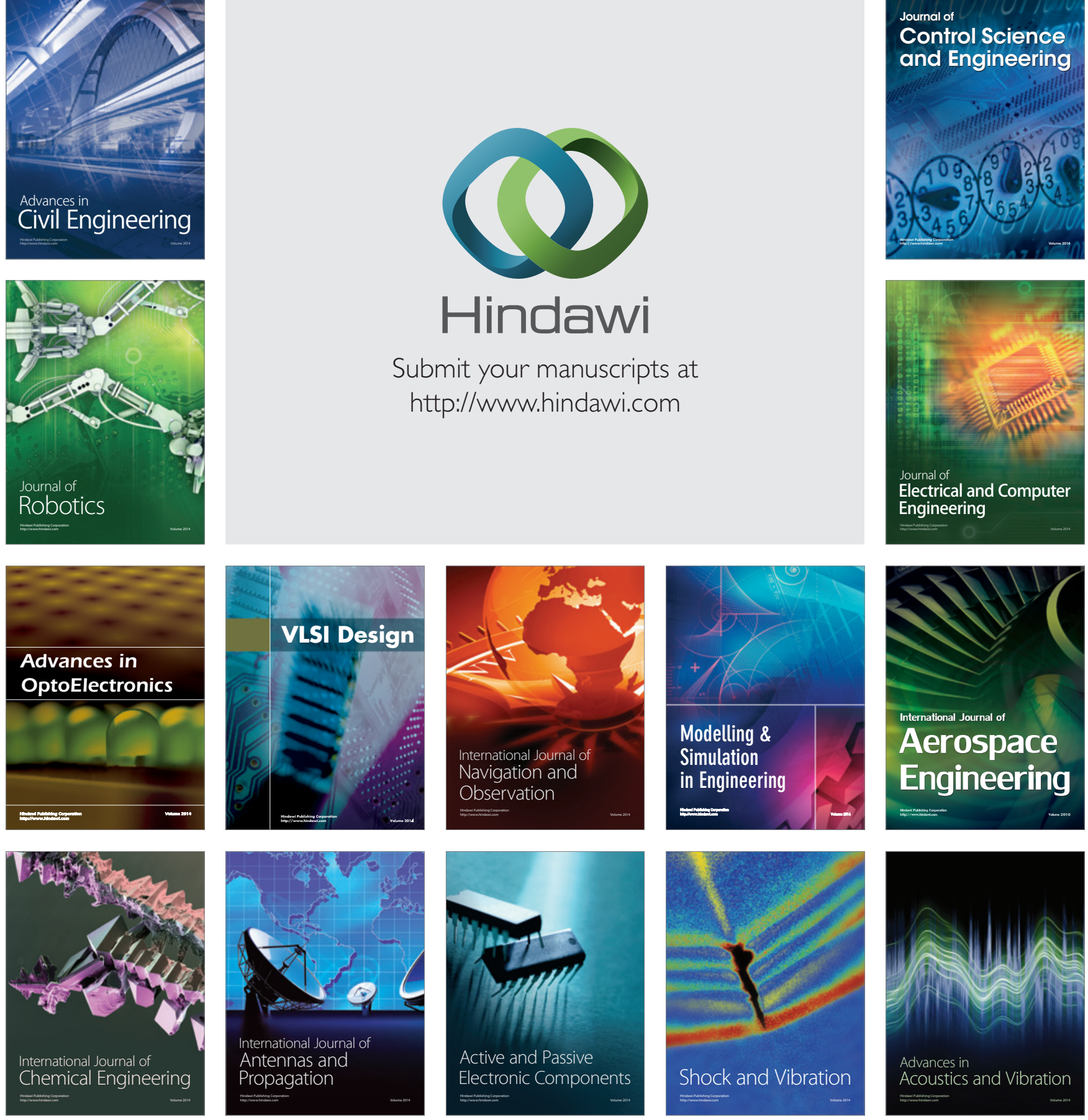